\title{
Exploiting Localization for Faster Power System Dynamic Simulations
}

\author{
Petros Aristidou \\ Department of Electrical Engineering \\ and Computer Science \\ University of Liège \\ Liège, Belgium \\ p.aristidou@ieee.org
}

\author{
Davide Fabozzi \\ Department of Chemical Engineering \\ Imperial College London \\ London, UK
}

\author{
Thierry Van Cutsem \\ Fund for Scientific Research (FNRS) at \\ Department of Electrical Engineering \\ and Computer Science \\ University of Liège \\ Liège, Belgium \\ t.vancutsem@ulg.ac.be
}

\begin{abstract}
This paper proposes an algorithm for exploiting the localized response of power system components to accelerate dynamic simulations. During the simulation, components marginally participating to the system dynamics are characterized as latent and their dynamic models are replaced by much simpler equivalents. At the same time, components with significant dynamic activity are characterized as active and their original dynamic models are used. Based on the criterion proposed, components switch status between active and latent to increase performance while retaining accuracy. Two realistic test systems, a medium-scale and a large-scale, are used for the performance evaluation of the proposed method.

Index Terms - differential-algebraic equations, dynamic simulation, Newton method, latency, localization, digital signal processing
\end{abstract}

\section{INTRODUCTION}

Dynamic simulations are used in industry and academia to check the response of electric power systems to various disturbances. They find application in simulator-based design of new control schemes and components, operator training in control centers, dynamic security assessment, hardware/software-inthe-loop studies, etc.

Dynamic power system models represent complex electric equipment (generators, motors, loads, wind turbines, etc.) that interact through the network. Usually, the equipment models are described by non-linear, stiff and hybrid Differential and Algebraic Equations (DAEs), while the network by linear algebraic equations [1]. A large interconnected power system may involve hundreds of thousands of DAEs spanning over different time scales and undergoing many discrete transitions imposed by limiters, switching devices, etc. Due to the huge size and complexity of these models, dynamic simulations are often extremely time consuming.

In the last decades, the speed of dynamic simulations has improved, mainly due to the performance of computing equipment and the development of new simulation algorithms. However, for many time-critical security applications the performance of dynamic simulations poses a serious obstacle. As a result, many security analysis softwares resort to static or quasi-steady-state simulations which are faster but use simplified dynamic or static models [2]. Their main drawback is that the model simplification is performed beforehand and cannot be changed during the simulation. Furthermore, a universal equivalent that would be valid for all power system dynamic phenomena, to the authors' knowledge, is still to be found.

In this paper we exploit the localized nature of power system response to a disturbance to provide faster dynamic simulations while preserving accuracy. The concept of localization results from the observation that in large power systems a disturbance affects a small number of components while the remaining are unaffected or slightly influenced [3]. Similar observations have been made in other fields [4].

In brief, during the simulation the proposed algorithm detects the components marginally participating to the system dynamics (latent) and replaces their dynamic models with much simpler and faster to compute equivalents. At the same time, the full dynamic model is used if the algorithm detects that a component exhibits significant dynamic activity (active). The algorithm employs simple and fast to compute metrics to classify each component into active or latent. Finally, the classification criterion can be tuned to obtain the desired balance between accuracy and speed.

The paper is organized as follows: in Section II we present the dynamic modeling of the power system. In Section III, we explain the derivation of the equivalent model used to replace latent components. In Section IV, we introduce the metrics and the criterion used for the categorization of the components. The simulation results are reported in Sections $\mathrm{V}$ and VI, followed by closing remarks in Section VII.

\section{Dynamic Modeling of Components}

Let the power system be decomposed into the network and a number of components, as sketched in Fig. 1. For reasons of simplicity, all components connected to the network, producing or consuming power are called injectors.

On the one hand, each injector $i$ is described by a system of non-linear Differential-Algebraic Equations (DAE) [1]:

$$
\boldsymbol{\Gamma}_{i} \dot{\boldsymbol{x}}_{i}=\boldsymbol{\Phi}_{i}\left(\boldsymbol{x}_{i}, \boldsymbol{V}\right)
$$

where $\boldsymbol{V}$ is the vector of network voltages, $\boldsymbol{x}_{i}$ is the state vector containing differential and algebraic variables of the 


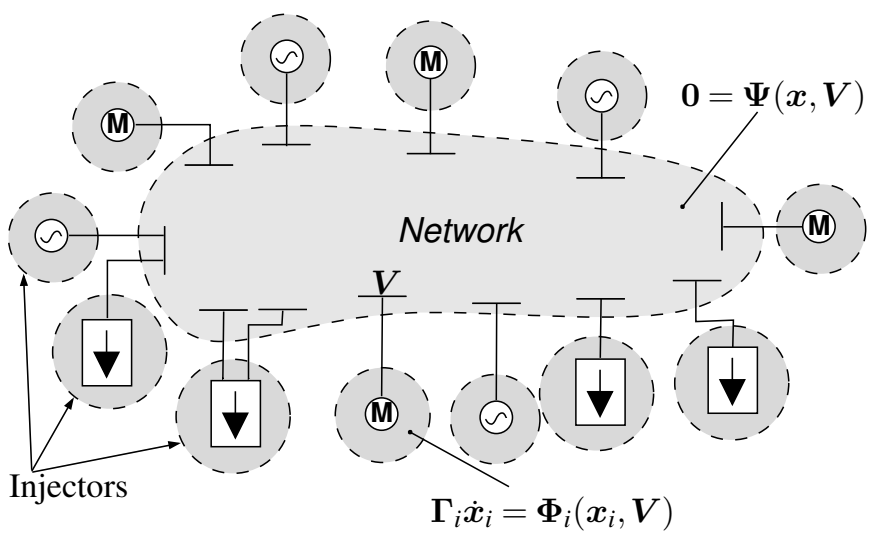

Figure 1. Decomposed Power System

$i$-th injector and $\boldsymbol{\Gamma}_{i}$ is a diagonal matrix with

$$
\left(\boldsymbol{\Gamma}_{i}\right)_{\ell \ell}= \begin{cases}0 & \text { if the } \ell \text {-th equation is algebraic } \\ 1 & \text { if the } \ell \text {-th equation is differential. }\end{cases}
$$

The current variables of the $i$-th injector introduced in the network relate to its states through:

$$
\boldsymbol{I}_{i}=\boldsymbol{C}_{i} \boldsymbol{x}_{i}
$$

where $C_{i}$ is simply a matrix selecting the current $\left(\boldsymbol{I}_{i}\right)$ from within the extended state vector $\boldsymbol{x}_{i}$.

On the other hand, the linear algebraic network equations are described by:

$$
\mathbf{0}=\boldsymbol{D} \boldsymbol{V}-\boldsymbol{I}=\boldsymbol{D} \boldsymbol{V}-\sum_{i=1}^{n} \boldsymbol{C}_{i} \boldsymbol{x}_{i}=\boldsymbol{g}(\boldsymbol{x}, \boldsymbol{V})
$$

For the purpose of numerical simulation, the injector DAE systems (1) are algebraized using a differentiation formula (such as Trapezoidal Rule, Backward Differentiation Formula, etc.) to get the corresponding non-linear algebraized systems:

$$
\mathbf{0}=\boldsymbol{f}_{i}\left(\boldsymbol{x}_{i}, \boldsymbol{V}\right), \quad i=1, \ldots, n .
$$

At each discrete time-step $t_{n}$ the non-linear algebraized injector equations (4) are solved together with the network equations (3) using a Newton method to compute the state vectors $\boldsymbol{x}\left(t_{n}\right)$ and $\boldsymbol{V}\left(t_{n}\right)$. At the $k$-th Newton iteration, the linearized injector systems have to be solved simultaneously with the linear network equations $(i=1, \ldots, n)$ :

$$
\begin{gathered}
\boldsymbol{A}_{i} \Delta \boldsymbol{x}_{i}+\boldsymbol{B}_{i} \Delta \boldsymbol{V}=-\boldsymbol{f}_{i}\left(\boldsymbol{x}_{i}^{k-1}, \boldsymbol{V}^{k-1}\right) \\
\boldsymbol{D} \Delta \boldsymbol{V}-\sum_{i=1}^{n} \boldsymbol{C}_{i} \Delta \boldsymbol{x}_{i}=-\boldsymbol{g}\left(\boldsymbol{x}^{k-1}, \boldsymbol{V}^{k-1}\right)
\end{gathered}
$$

The solution is computed using the decomposed and accelerated Newton scheme [5]. In brief, the injector equations (5) are solved with respect to $\Delta \boldsymbol{x}_{i}$ and is introduced in (6) to obtain the following reduced system:

$$
\begin{aligned}
\left(\boldsymbol{D}+\sum_{i=1}^{n} \boldsymbol{C}_{i} \boldsymbol{A}_{i}^{-1} \boldsymbol{B}_{i}\right) \Delta \boldsymbol{V}= & -\boldsymbol{g}\left(\boldsymbol{x}^{k-1}, \boldsymbol{V}^{k-1}\right) \\
& -\sum_{i=1}^{n} \boldsymbol{C}_{i} \boldsymbol{A}_{i}^{-1} \boldsymbol{f}_{i}\left(\boldsymbol{x}_{i}^{k-1}, \boldsymbol{V}^{k-1}\right)
\end{aligned}
$$

This reduced system is solved to obtain the voltage correction $\Delta \boldsymbol{V}$ which is backward substituted in (5) to get the state corrections $\Delta \boldsymbol{x}_{i}$.

While this decomposition method is numerically equivalent to an integrated Newton scheme applied on equations (3) and (4), it provides access to the individual injector models and allows their separate treatment [6]. This feature is exploited by the localization algorithm to switch between full and simplified models during the simulation without expensive operations related to matrix computing and factorizing.

\section{Equivalent Model of Latent InJectors}

When an injector is declared latent, its detailed dynamic model (1) is replaced by a smaller and faster to compute linear model. This is derived from the linearized equations (5) when ignoring the internal dynamics, that is $\boldsymbol{f}_{i}\left(\boldsymbol{x}_{i}^{k-1}, \boldsymbol{V}^{k-1}\right) \simeq \mathbf{0}$, and solving for the state variation $\Delta \boldsymbol{x}_{i}$ :

$$
\Delta \boldsymbol{x}_{i} \simeq-\boldsymbol{A}_{i}^{-1} \boldsymbol{B}_{i} \Delta \boldsymbol{V}
$$

The corresponding current variation is obtained from (2) as:

$$
\Delta \boldsymbol{I}_{i}=-\boldsymbol{C}_{i} \boldsymbol{A}_{i}^{-1} \boldsymbol{B}_{i} \Delta \boldsymbol{V}=-\boldsymbol{G}_{i} \Delta \boldsymbol{V}
$$

where $\boldsymbol{G}_{i}$ is a sensitivity matrix relating the current $\left(\Delta \boldsymbol{I}_{i}\right)$ with the voltage $(\Delta \boldsymbol{V})$ variation.

Selecting an arbitrary instant $t^{*}$, the linear relation (8) can be rewritten as:

$$
\boldsymbol{I}_{i}\left(t_{n}\right)=\boldsymbol{I}_{i}\left(t^{*}\right)-\boldsymbol{G}_{i}\left(t^{*}\right)\left[\boldsymbol{V}\left(t_{n}\right)-\boldsymbol{V}\left(t^{*}\right)\right]
$$

for any discrete time $t_{n} \geq t^{*}$.

The linear model (9) is a valid estimate of the full dynamic model (1) when the injector shows low dynamic activity (thus the term $\boldsymbol{f}_{i}\left(\boldsymbol{x}_{i}^{k-1}, \boldsymbol{V}^{k-1}\right)$ in (5) can be ignored) and only for small deviations around the linearization point (thus $\boldsymbol{G}_{i}$ can be considered constant).

\section{LOCALIZATION AlgorithM}

The essence of the algorithm lies in its ability to detect the status switching of injectors. During the dynamic simulation the state vector values $\boldsymbol{x}(t)$ and $\boldsymbol{V}(t)$ are known for $t \leq t_{n}$, with $t_{n}$ the last computed discrete time. The injector classification criteria have to be robust and based only on currently available information. Furthermore, as the algorithm aims for higher simulation performance, the criteria computations need to be fast and use as little memory as possible.

\section{A. Monitoring Variable and Metrics}

Since the injectors interact with the network and between them through the current and voltage changes (see Eq. 3), power flow variations can be used as an indication of dynamic activity. Therefore, the variation of the apparent power $\left(S_{i}=\sqrt{P_{i}{ }^{2}+Q_{i}{ }^{2}}\right)$ flowing in each injector was naturally selected as the monitoring variable representative to the injector dynamic activity. Alternatively, more detailed information could be extracted from the active and reactive powers but at the cost of doubling the computing effort and memory usage. 
Simply stated, an injector is declared latent when its apparent power $S_{i}$ has "not changed significantly for some time" or, in other words, exhibits small variability. The $S_{i}$ values are available as time-series samples. Thus, traditional methods for analyzing time series data can be employed to characterize the variability of $S_{i}$ over a pre-specified, moving, time window.

The choice of using a moving time window and not the entire history aims at disregarding the oldest "behavior" of an injector and involving only recently observed dynamics. On the other hand, if the time window is very small, smooth variations may not be detected.

The main characteristics extracted from the time series are the sample average value and variance (or standard deviation). In particular, the standard deviation is the measure of volatility that shows how much variation or dispersion exists from the average. A small standard deviation indicates that the data points tend to be very close to the average, whereas high standard deviation indicates that the data points are spread out over a large range of values. Consequently, a small standard deviation of $S_{i}$ is an indication that the $i$-th injector exhibits low dynamic activity and can be considered latent.

After an injector is classified as latent at time $t^{*}$, the apparent power is no longer dictated by the dynamic model (1) but by the linear model (9), affected only by the deviation of the voltage. Therefore, we cannot rely on the standard deviation to switch the injector back to active mode since slow voltage changes can gradually "drift" the injector's operating point away from the reference without the standard deviation ever increasing. To avoid this, we monitor the absolute deviation of the apparent power from its reference value $S_{i}\left(t^{*}\right)$. If the absolute variation is bigger than a tolerance $\epsilon_{L}$, meaning that the model has moved away from the linearization point, the injector returns to active mode.

\section{B. Signal Processing}

Large power systems may involve thousands of injectors, making it inefficient to keep all injector apparent powers in memory over the moving time window. Furthermore, calculating the exact average and standard deviation values of the timewindow samples at each time-step leads to complex bookkeeping and time consuming computations. To avoid this, an approximation is considered, which originates from realtime digital signal processing where computing and memory resources are scarce [7]. Its applications include audio/video processing, speech recognition, embedded systems, etc.

When a new sample $S_{i}\left(t_{n}\right)$ is computed, the average value is obtained as the weighted sum of the new sample and the previous average value $S_{i, a v}\left(t_{n-1}\right)$ according to:

$$
S_{i, a v}\left(t_{n}\right)=\left(1-\lambda_{1}\right) * S_{i, a v}\left(t_{n-1}\right)+\lambda_{1} * S_{i}\left(t_{n}\right)
$$

where $\lambda_{1}$ is a "forgetting factor" with $0 \leq \lambda_{1} \leq 1$.

Next, the difference between the new value and the computed average, i.e. $\triangle S_{i}\left(t_{n}\right)=S_{i}\left(t_{n}\right)-S_{i, a v}\left(t_{n}\right)$, is used to calculate the approximate variance:

$$
S_{i, v a r}\left(t_{n}\right)=\left(1-\lambda_{2}\right) * S_{i, v a r}\left(t_{n-1}\right)+\lambda_{2} * \triangle S_{i}\left(t_{n}\right)^{2}
$$

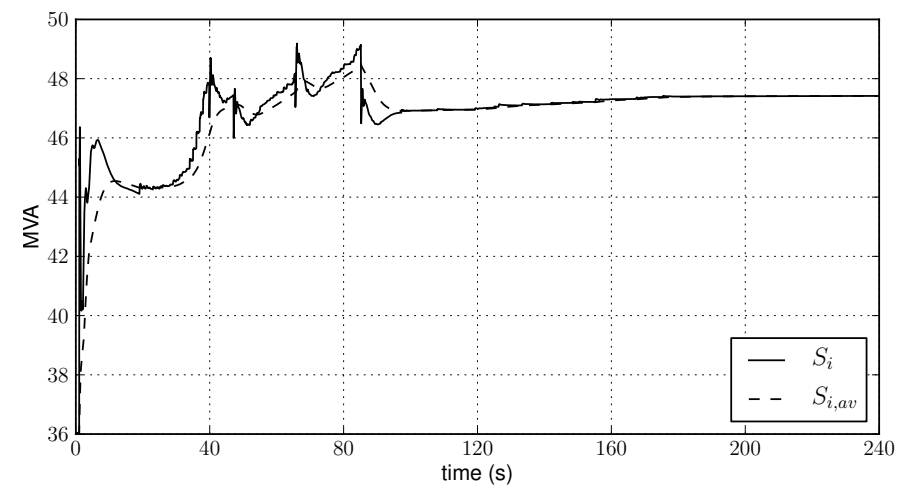

Figure 2. Actual and average apparent power

with $0 \leq \lambda_{2} \leq 1$.

Finally, the standard deviation is obtained as:

$$
S_{i, s t d}\left(t_{n}\right)=\sqrt{S_{i, v a r}\left(t_{n}\right)}
$$

and must be compared to a pre-defined threshold $\epsilon_{L} \geq 0$ to decide whether the injector is latent or not.

This procedure is equivalent to passing the input digital signal $S_{i}\left(t_{n}\right)$ through a single-pole DC blocking high-pass filter (with a pole at $\lambda_{1}$ ) and then sending the resulting signal to a root mean square detector (whose response time is set by $\lambda_{2}$ ) to measure the signal's variability once its central trend has been removed [8].

Tuning the latency criterion requires setting the values of $\lambda_{1}, \lambda_{2}$ and $\epsilon_{L}$. The parameter $\lambda_{1}$ sets the time window of observation for averaging. That is, a smaller $\lambda_{1}$ value extends the observation window while a larger curtails it. The parameter $\lambda_{2}$ sets the response time to the changes $\triangle S_{i}$, that is, a smaller $\lambda_{2}$ value makes the criterion less responsive. Finally, the parameter $\epsilon_{L}$ is the allowed tolerance (in MVA) for classifying the injector $\left(\epsilon_{L}=0\right.$ results in fully accurate simulation).

The three parameters have to be tuned for the system of concern. However, once tuned, they can be used for any simulated disturbance. In particular, $\lambda_{1}$ and $\lambda_{2}$ depend on the time step size chosen for the simulation which is equal to the time interval between two samples. If a larger time step size than the one used for tuning is selected, the observation window and response time increase, leading to a more conservative criterion. Thus, it has been found appropriate to tune $\lambda_{1}$ and $\lambda_{2}$ for the smallest time-step used and keep them constant. The selection of the last parameter $\epsilon_{L}$ relies mainly on the approximation we are disposed to accept. If the power system includes injectors of both very small and very large powers then $\epsilon_{L}$ must remain small to keep the error bounded. On the other hand, if the system involves similarly sized injectors, then $\epsilon_{L}$ can be increased without introducing big errors.

\section{Illustrative Example}

An example of the monitoring variable $S_{i}$ of an injector after a disturbance can be seen in Fig. 2. In the same figure its average calculated using Eq. (10) with $\lambda_{1}=0.01$ is displayed. 


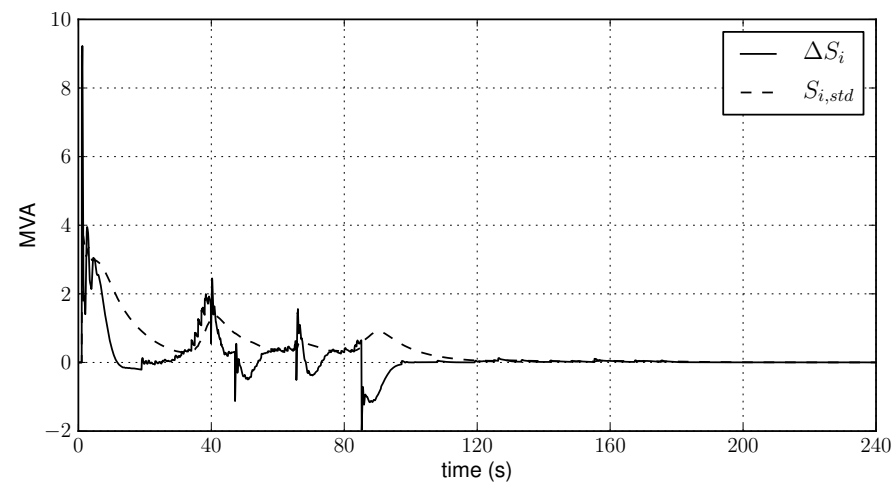

Figure 3. Difference and Standard Deviation

Figure 3 shows the difference $\triangle S_{i}$ and the standard deviation calculated using Eq. (12) with $\lambda_{2}=0.01$.

\section{Switching Algorithm}

The decision for switching between active and latent mode is taken after computing each time step $t_{n}$. Then, the selected models are used for the computation of the states at $t_{n+1}$. During the Newton iterations, the models chosen for latent and active injectors are kept unchanged as switching models could perturb the iterations and cause divergence.

The complete procedure is given by Algorithm 1. This algorithm was implemented in the academic simulation software RAMSES, developed at the University of Liège.

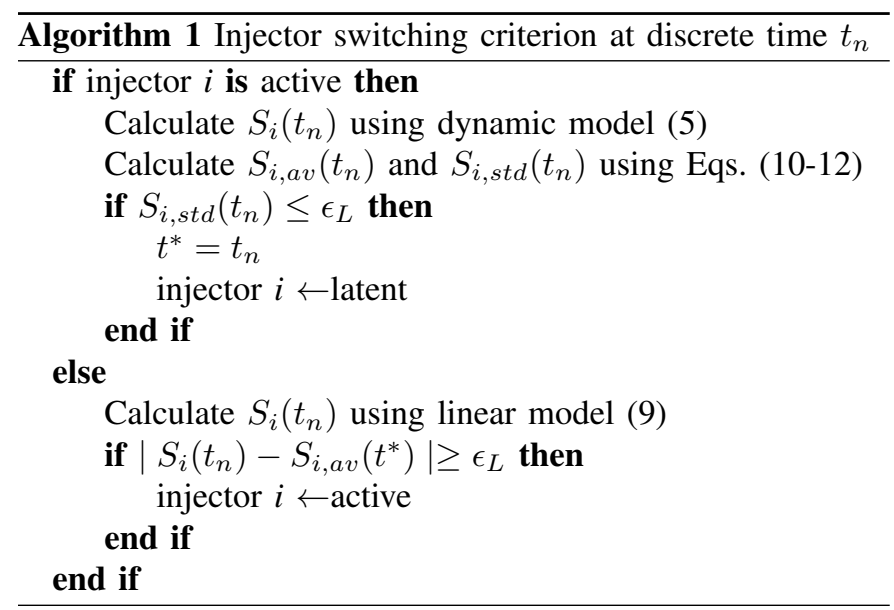

\section{E. Early Simulation Halting}

When a dynamic response is stable, the system reaches a steady state after all dynamics have faded. As the system approaches steady state, more and more injectors exhibit low dynamic activity and switch to latent. If at some point all injectors become latent, the simulation will involve only the linear algebraic systems (3) and (9), thus, no further dynamic activity can be observed.

This feature can be exploited to avoid unnecessary computations when the system has already reached steady state. For example, it can find application in dynamic security assessment as an early stopping criterion of stable simulations.

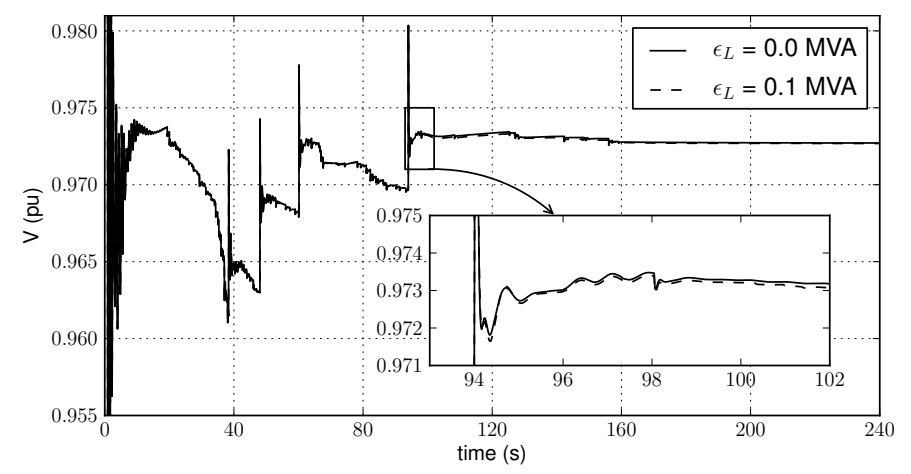

Figure 4. 2204-bus system: Voltage evolution on bus near fault

Table I

RESULTS WITH THE 2204-BUS SYSTEM

\begin{tabular}{|c|c|c|c|c|}
\hline $\begin{array}{c}\epsilon_{L} \\
(\mathrm{MVA})\end{array}$ & $\begin{array}{c}\text { Execution Time } \\
(\mathrm{s})\end{array}$ & $\begin{array}{c}\text { Speedup } \\
(\text { times })\end{array}$ & $\begin{array}{c}\text { Aver. } V_{\text {err }} \\
(\mathrm{pu})\end{array}$ & $\begin{array}{c}\text { Aver. } S_{i, e r r} \\
(\%)\end{array}$ \\
\hline \hline 0.0 & 77 & - & - & - \\
\hline 0.1 & 55 & 1.41 & $3 \mathrm{E}-5$ & 0.09 \\
\hline 0.5 & 46 & 1.69 & $3 \mathrm{E}-4$ & 0.21 \\
\hline 1.0 & 39 & 1.96 & $5 \mathrm{E}-4$ & 0.48 \\
\hline
\end{tabular}

\section{Results With THE 2204-BUS SYSTEM}

This section reports on results obtained with the mediumsize model including 2204 buses, 2919 branches and 135 power plants with a detailed representation of the synchronous machine, its excitation system, automatic voltage regulator, power system stabilizer, turbine and speed governor. The model also includes 976 dynamically modeled loads. The resulting DAE system has 11774 states.

The disturbance consists of a short circuit lasting 7 cycles, that is cleared by opening a line. The system is simulated over a period of $240 \mathrm{~s}$ with a time step size of 1 cycle. The system evolves in the long term under the effect of 1076 load tap changers, 24 automatic shunt compensation switching devices as well as overexcitation limiters. The same simulation was performed with four different values of the latency tolerance $\epsilon_{L}$ while keeping the other parameters $\left(\lambda_{1}=0.001, \lambda_{2}=\right.$ 0.01 ) unchanged.

As discussed in Section IV-B, $\lambda_{1}$ was tuned to provide a moving averaging window of approximately 1000 cycles. Similarly, $\lambda_{2}$ was tuned with a response time of approximately 100 cycles. The values chosen offer a robust but not very nervous variability detection. Finally, $\epsilon_{L}$ was varied between 0 and 1 MVA to show its effect on speed and accuracy.

Table I shows the speedup and average error from using the localization scheme. For a conservative tolerance of $\epsilon_{L}=0.1$ MVA the simulation runs 1.41 times faster. At the same time, the average voltage error at the bus nearest to the fault is $3 E-05 \mathrm{pu}$ and for the apparent power of a power plant near the fault the average error is $09 \%$. As foretold, increasing the tolerance leads to higher simulation performance (up to 1.96 times faster) but bigger errors. Still, even with a large tolerance of $\epsilon_{L}=1$ MVA the simulation accuracy is very good.

Figures 4 and 5 show the voltage evolution at the bus nearest 


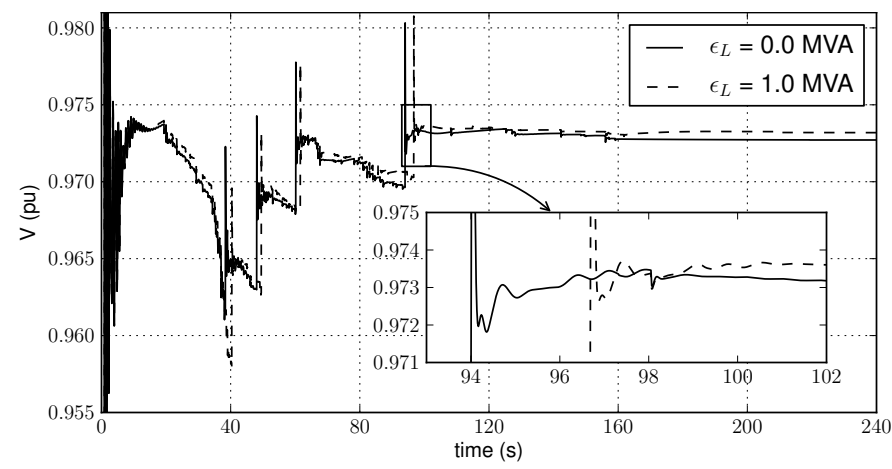

Figure 5. 2204-bus system: Voltage evolution on bus near fault

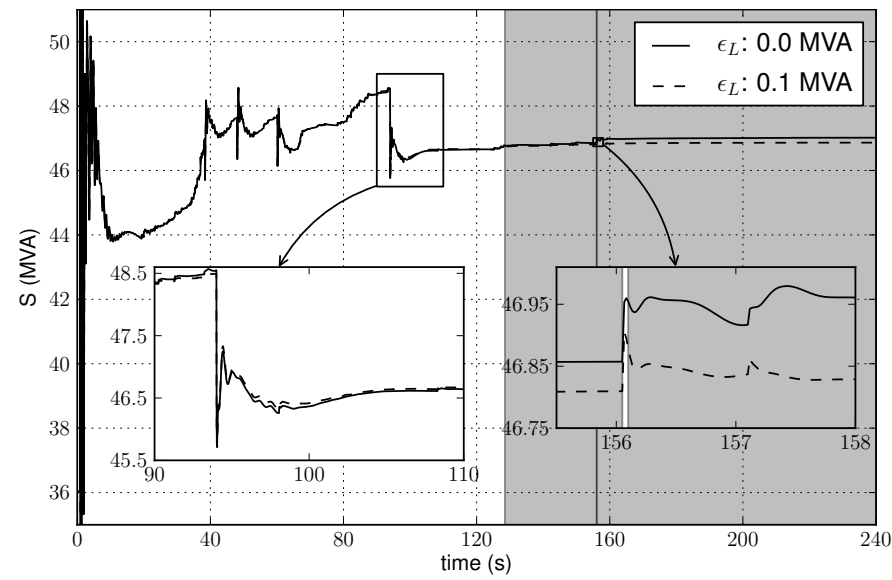

Figure 6. 2204-bus system: Evolution of synchronous machine apparent power (latent mode shown in gray)

to the fault with and without localization for two different tolerance levels. In the first figure, the two evolutions are for every practical application indiscernible with a maximum error of $0.0003 \mathrm{pu}$ appearing around $t=94 \mathrm{~s}$ (see zoom in Fig. 4). This error coincides with the switching of a large shunt reactor by an automatic device responding to the voltage drops. The switching time is shifted by a few milliseconds causing the appearance of the maximum error. These devices, like load tap changers, are discrete devices with response times in the order of 10 seconds. Therefore, a shifted by a few milliseconds switching has no practical consequence. In the second figure, the error is increased because of the higher tolerance but nevertheless the average error remains very small.

Likewise, Figs. 6 and 8 show the apparent power of a medium-size power plant close to the fault. The power plant is identified as latent (i.e. its full model is replaced by the sensitivity model) in the time intervals shown in gray and active in the rest. The vertical black lines show the transitions between modes. More specifically, the plant gets back to active mode once for a short period of time at $t=156 \mathrm{~s}$ (see zoom in Fig. 6). In the second figure the injector gets latent faster because of the higher latency tolerant. As with the voltage, the bigger absolute error in both figures coincides with shifted in time automatic shunt compensation switching.

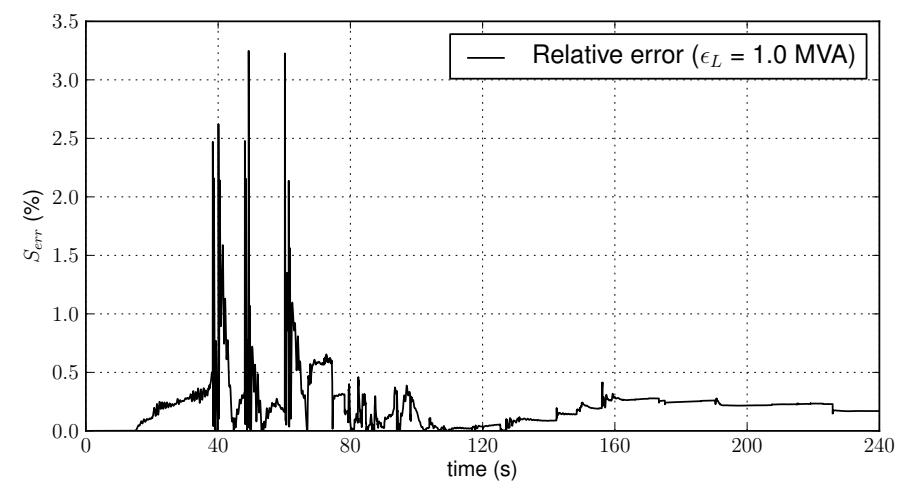

Figure 7. 2204-bus system: Relative error of apparent power

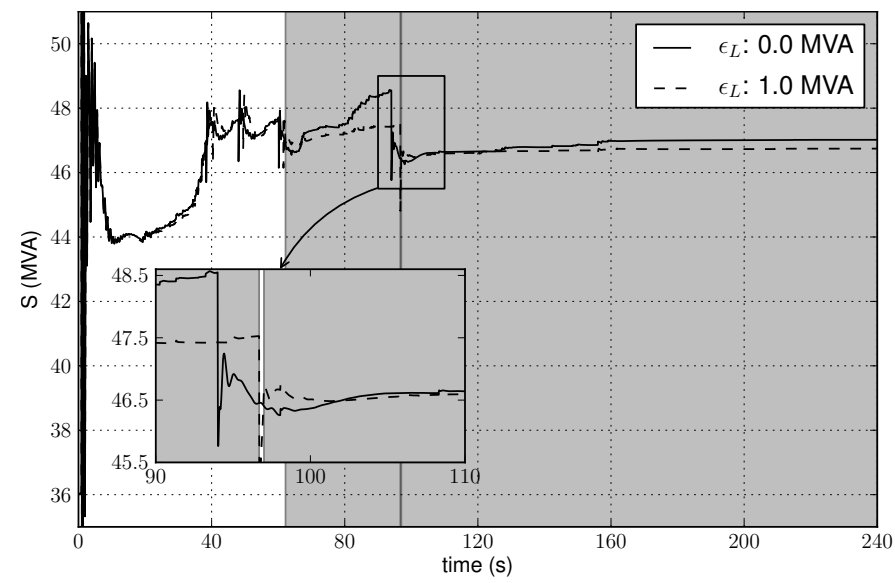

Figure 8. 2204-bus system: Evolution of synchronous machine apparent power (latent mode shown in gray)

During the short-term dynamics and up to $20 \mathrm{~s}$ the localization criterion correctly detects the high dynamic activity in the system and keeps full accuracy. This can be easily seen in Fig. 7 with zero error in this period. As the injectors located away from the fault start becoming latent, some error is introduced with maxima (shown as peaks in Fig. 7) appearing at shunt compensation switching times. The maximum state deviation appears in components closer to the disturbance area. The reason for this is that these, more active components, accumulate the small errors introduced by the injectors already latent. More advanced algorithms [9] can be used to quantify the overall deviation but are very hard to compute when the system under consideration involves tens or even hundreds of thousands of states.

As discussed in Section IV-E, the algorithm can detect the reaching of steady state and the lack of dynamic activity and signal an early termination. In fact, for the same simulated disturbance with $\epsilon_{L}=0.1 \mathrm{MVA}$, if the simulation time is increased to $300 \mathrm{~s}$ it is observed that all injectors become latent around $283 \mathrm{~s}$. At that moment the simulation is halted.

\section{RESUlTS With THE 15226-BUS SYSTEM}

This section reports on results obtained with the large-scale system described in [5], set up in the context of the FP7 


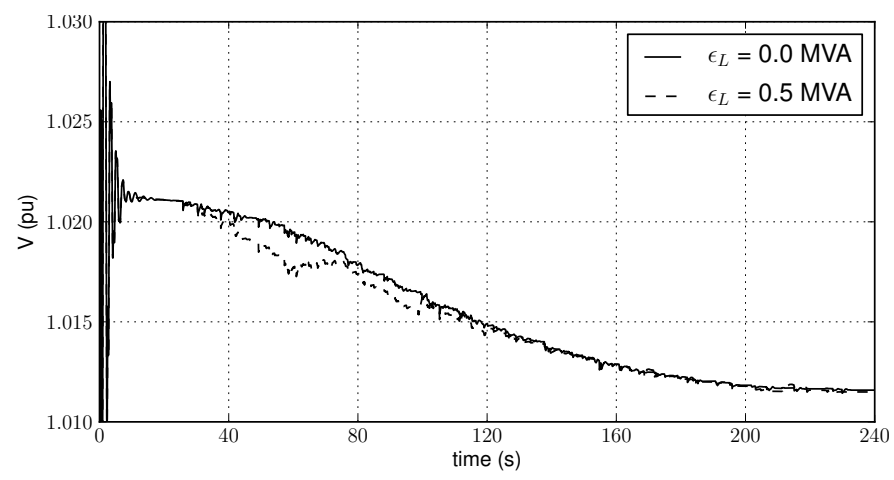

Figure 9. 15226-bus system: Voltage evolution on bus near fault

Table II

RESULTS WITH THE 15226-BUS SYSTEM

\begin{tabular}{|c|c|c|c|c|}
\hline $\begin{array}{c}\epsilon_{L} \\
(\mathrm{MVA})\end{array}$ & $\begin{array}{c}\text { Execution Time } \\
(\mathrm{s})\end{array}$ & $\begin{array}{c}\text { Speedup } \\
\text { (times) }\end{array}$ & $\begin{array}{c}\text { Aver. } V_{\text {err }} \\
(\mathrm{pu})\end{array}$ & $\begin{array}{c}\text { Aver. } S_{i, e r r} \\
(\%)\end{array}$ \\
\hline \hline 0.0 & 806 & - & - & - \\
\hline 0.1 & 300 & 2.69 & $5 \mathrm{E}-5$ & 0.01 \\
\hline 0.5 & 246 & 3.28 & $3 \mathrm{E}-4$ & 0.05 \\
\hline 1.0 & 222 & 3.63 & $1 \mathrm{E}-3$ & 0.21 \\
\hline
\end{tabular}

European PEGASE project [10]. The model includes 15226 buses, 21765 branches and 3483 power plants with a detailed representation of each synchronous machine, its excitation system, automatic voltage regulator, power system stabilizer, turbine and speed governor. The model also includes 7211 dynamically modeled loads. The resulting DAE system has 146239 states.

The disturbance consists of a short circuit lasting 5 cycles, that is cleared by opening two double-circuit lines. The system is simulated over a period of $240 \mathrm{~s}$ with a time step size of 1 cycle. The system evolves in the long term under the effect of load tap changers as well as overexcitation limiters. The same simulation was performed with four different values of latency tolerance $\epsilon_{L}$ while keeping the other parameters unchanged.

Table II shows the speedup and average error from using the localization scheme. For a conservative tolerance of $\epsilon_{L}=0.1$ MVA the simulation runs 2.69 times faster. At the same time, the average voltage error at the bus nearest to the fault is $5 E-05 \mathrm{pu}$ and for the apparent power of a power plant near the fault the average error is $0.01 \%$. As foretold, increasing the tolerance leads to higher simulation performance (up to 3.63 times faster) but bigger errors. Still, even with a large tolerance of $\epsilon_{L}=1$ MVA the simulation accuracy is very good. Figure 9 shows the voltage evolution at the bus nearest to the fault with and without localization and Fig. 10 the corresponding absolute error between them. The average error is small with spikes appearing close to shifted in time load tap changes.

It has to be noted that the large-scale system benefits more from the localization algorithm in terms of performance. This is to be expected as larger interconnected systems exhibit a comparatively more localized response to disturbances. The effects usually do not propagate to remote parts but if they do, the proposed algorithm would automatically switch the

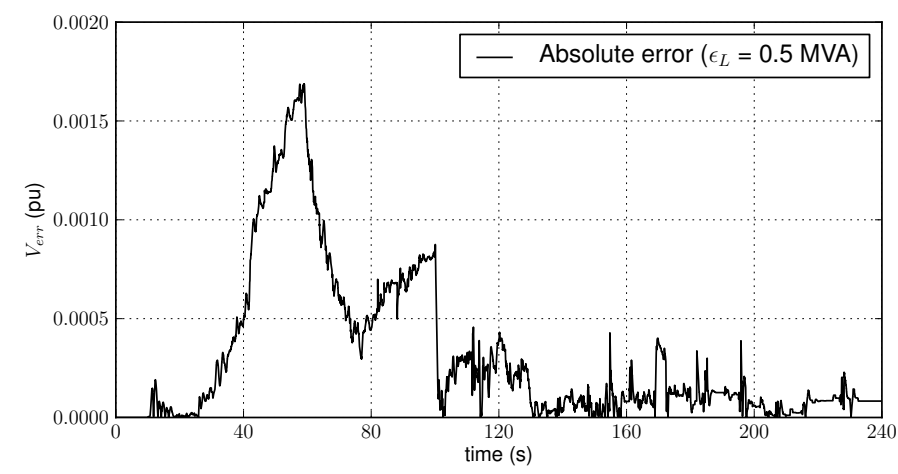

Figure 10. 15226-bus system: Absolute error of voltage

involved components back from latent to active.

\section{CONCLUSION}

An algorithm has been proposed to exploit localization and accelerate dynamic simulations. This is done by targeted (based on the dynamic activity) substitution of injector dynamic models with smaller and faster to compute equivalents. The error introduced by the equivalents is bounded and can be easily controlled, through the latency tolerance $\epsilon_{L}$, to balance desired speed and accuracy. The method was tested on two power system models yielding significant simulation speedup with a minor effect on accuracy.

As a result, the localization algorithm can be used in dynamic security assessment to provide higher performance both from the simulation speedup as well as its ability to identify when the system reaches steady state and halt the simulation. It can be easily combined with parallelized contingency simulations to shorten the overall computing time.

\section{REFERENCES}

[1] P. Kundur, Power system stability and control. McGraw-hill New York, 1994.

[2] U. D. Annakkage, N. K. C. Nair, Y. Liang, A. M. Gole, V. Dinavahi, B. Gustavsen, T. Noda, H. Ghasemi, A. Monti, M. Matar, R. Iravani, and J. A. Martinez, "Dynamic System Equivalents: A Survey of Available Techniques," IEEE Trans. Power Delivery, vol. 27, no. 1, pp. 411-420, Jan. 2012.

[3] V. Brandwajn, "Localization Concepts in (In)-Security Analysis," in Proc. 1993 Athens Power Tech Conf., vol. 1, Sept. 1993, pp. 10-15.

[4] G. D. Hachtel and A. L. Sangiovanni-Vincentelli, "A survey of thirdgeneration simulation techniques," Proceedings of the IEEE, vol. 69, no. 10, pp. 1264-1280, Oct. 1981.

[5] D. Fabozzi, "Decomposition, Localization and Time-Averaging Approaches in Large-Scale Power System Dynamic Simulation," Ph.D. dissertation, University of Liège, 2012. [Online]. Available: http://hdl.handle.net/2268/126720

[6] D. Fabozzi and T. Van Cutsem, "Localization and latency concepts applied to time simulation of large power systems," in Bulk Power System Dynamics and Control (iREP)-VIII (iREP), 2010 iREP Symposium. IEEE, 2010, pp. 1-14.

[7] A. Oppenheim, A. Willsky, and S. Nawab, Signals and systems, ser. Prentice-Hall signal processing series. Prentice Hall, 1997.

[8] S. Kuo, B. Lee, and W. Tian, Real-Time Digital Signal Processing: Implementations and Applications. John Wiley \& Sons, 2006.

[9] D. Fabozzi and T. Van Cutsem, "Assessing the proximity of time evolutions through dynamic time warping," Generation, Transmission Distribution, IET, vol. 5, no. 12, pp. 1268 -1276, december 2011.

[10] (2013, January) Pan european grid advanced simulation and state estimation (PEGASE). [Online]. Available: http://www.fp7-pegase.eu/ 